\title{
Clinical experience with pegaptanib sodium
}

\author{
Chiara Rosina \\ Ferdinando Bottoni \\ Giovanni Staurenghi \\ Luigi Sacco Hospital, University of \\ Milano, Italy
}

\begin{abstract}
Pegaptanib sodium (Macugen ${ }^{\circledR}$ ) blocks the extracellular vascular endothelial growth factor (VEGF) isoform VEGF $_{165}$, whose elevated levels are associated with the development of choroidal neovascularization (CNV). This selective inhibition prevents binding to the VEGF receptors and the development of the increased vascular permeability and the CNV associated with neovascular age-related degeneration (AMD). The VEGF Inhibition Study In Ocular Neovascularization (VISION) demonstrated that pegaptanib sodium confers clinically meaningful benefit in the treatment of all angiographic subtypes of neovascular AMD. It also has a favorable safety profile after 1 and 2 years of continuous treatment, and recent data suggest that the agent has a disease-modifying effect. Post hoc analysis of VISION suggests that treatment benefit may be greatest in patients with early lesions, in whom $80 \%$ achieved the primary endpoint of $<15$ letters lost, $47 \%$ maintained visual acuity (VA), and $20 \%$ gained $\geq 15$ letters of vision. Similarly, our own clinical experience indicates that pegaptanib sodium achieves better outcomes in early lesions than in established lesions, particularly in patients with previously untreated minimally classic and occult lesions in whom VA improvement and lesion size stabilization has been recorded. Observations indicate that pegaptanib sodium has a slower mode of action than unselective VEGF inhibitors, resulting in an average of 3-4 injections being required to stabilize VA and lesion size. Pegaptanib sodium has good efficacy and safety profiles and represents a good treatment option for patients with early CNV membranes associated with neovascular AMD.
\end{abstract}

Keywords: vascular endothelial growth factor (VEGF), choroidal neovasularization (CNV), agerelated macular degeneration (AMD), pegaptanib sodium, early lesions, visual acuity (VA)

Vascular endothelial growth factor (VEGF) is a proangiogenic factor that plays a key role in the pathogenesis of neovascular age-related macular dengeneration (AMD). Elevated levels of the VEGF ${ }_{165}$ isoform lead to the development of choroidal neovascularization (CNV), the growth of fragile, new vessels in the macula that may cause exudation, and hemorrhages. Pegaptanib sodium (Macugen ${ }^{\circledR}$, [OSI] Eyetech Pharmaceuticals, Pfizer) blocks the extracellular $\mathrm{VEGF}_{165}$ isoform and inhibits its binding to the VEGF receptors. This action prevents the increased vascular permeability and choroidal neovascularization that is associated with neovascular AMD.

\section{Efficacy}

The results of the VEGF Inhibition Study In Ocular Neovascularization (VISION) have shown that pegaptanib sodium confers statistically significant, and clinically meaningful, benefit in the treatment of all angiographic subtypes of neovascular AMD (Gragoudas et al 2004; Gonzales 2005). The trials randomized patients to receive pegaptanib sodium $0.3 \mathrm{mg}, 1.0 \mathrm{mg}$, or $3.0 \mathrm{mg}$ intravitreal (ITV) injections or sham injections every 6 weeks. The primary end point was the proportion of patients losing fewer than 15 letters from baseline visual acuity (VA) at 54 weeks. This was achieved in $70 \%$ of patients receiving $0.3 \mathrm{mg}$ pegaptanib sodium, regardless of
Correspondence: Giovanni Staurenghi via Muratori 29, 20153 Milano, Italy

Email giovanni.staurenghi@unimi.it 
photodynamic therapy (PDT), compared with 55\% in the placebo patient group $(\mathrm{p}<0.001)$. No additional benefits, in terms of clinical efficacy or safety, were measured in the 1.0 $\mathrm{mg}$ and $3.0 \mathrm{mg}$ patient groups compared with those receiving the lower $0.3 \mathrm{mg}$ dose.

During the second year of the study, mean VA was stabilized in patients receiving $0.3 \mathrm{mg}$ pegaptanib sodium, while those receiving placebo injections experienced continued vision loss. At year 2, change in vision (recorded as letters observed) was approximately $50 \%$ better in patients receiving $0.3 \mathrm{mg}$ pegaptanib sodium than in those receiving placebo injections, irrespective of CNV lesion subtype (Chakravarthy et al 2006).

On the basis of these findings, pegaptanib sodium has been approved at a recommended dosage of $0.3 \mathrm{mg}$, administered as an ITV injection every 6 weeks, for the treatment of neovascular AMD.

The results of VISION appear less encouraging than those recorded in the early ranibizumab clinical trials - the ANti-VEGF antibody for the treatment of predominantly classic CHORoidal neovascularization in AMD (ANCHOR) trial and the Minimally classic/occult trial of the Anti-VEGF antibody Ranibizumab In the treatment of Neovascular AMD (MARINA) (Brown et al 2006; Rosenfeld et al 2006a, b). However, it is important to consider differences in the patient populations recruited for the studies: MARINA recruited patients with minimally classic or occult lesions with no classic $\mathrm{CNV}$, and ANCHOR recruited patients with predominantly classic CNV lesions. VISION, however, enrolled patients with all neovascular AMD lesion subtypes (25\% predominantly classic, $39 \%$ occult with no classic and $36 \%$ minimally classic).

\section{Safety}

Pegaptanib sodium has a favorable safety profile after 1 and 2 years of continuous treatment. The 2-year safety results demonstrated that all doses of pegaptanib were well tolerated et al (D'Amico et al 2006). The most common ocular adverse events were transient, mild to moderate in intensity, and attributed to the injection preparation and procedure. In year 1, serious injection-related complications included endophthalmitis (at a rate of $0.16 \%$ per injection). Most of the cases resulted from violations of the injection preparation protocol and the rate dropped to $0.07 \%$ in the second year after reinforced aseptic procedure. In general, systemic adverse events were relatively low and similar in the pegaptanib sodium and placebo patient groups (D'Amico et al 2006).

\section{Retrospective analysis}

Mills et al (2007) determined that the treatment benefits afforded by pegaptanib sodium are due to its disease modifying effect, an analysis of which has been proposed by Whitehouse et al (1998) and by McDermott et al (2002). The authors of the aforementioned papers analyzed data from the second year of VISION. After the initial 54-week treatment period, patients were re-randomized to either continue or discontinue treatment (in an unmasked fashion) for a further 48 weeks. The authors found that visual outcomes in patients who were randomized to discontinue treatment after 54 weeks and who were followed for a further 48 weeks, were significantly different to those in patients receiving placebo injections in terms of loss of 15 letters of vision (relative risk [RR] 0.70, $95 \%$ confidence interval $[\mathrm{CI}] 0.57-0.88 ; \mathrm{p}=0.002$ ), indicating that the treatment had a modifying effect on the disease (Mills et al 2007).

In the post hoc analysis of VISION, the treatment benefit seen in patients receiving $0.3 \mathrm{mg}$ pegaptanib sodium was particularly marked in those with early lesions; $80 \%$ of those with early lesions achieved the primary end point of $<15$ letters lost. In addition, nearly half $(47 \%)$ of patients with early lesions maintained VA when treated with $0.3 \mathrm{mg}$ pegaptanib sodium, and $20 \%$ of these patients gained $\geq 15$ letters of vision (Gonzales 2005).

\section{Clinical practice}

In our clinical centre, we treated 45 eyes (in 45 patients) with pegaptanib sodium, between June 2006 and June 2007. The lesion subtypes treated were: classic lesions in 5 eyes, minimally classic lesions in 7 eyes, and occult lesions in 33 eyes. Of these patients, 22 were followed for 18 weeks.

From our own clinical experience of treating all neovascular AMD lesion subtypes with pegaptanib sodium, the efficacy of the agent differs when comparing treatment outcomes in patients with early lesions and those with established lesions. In our department it has been observed that pegaptanib sodium treatment in early lesions results in better outcomes than in established lesions. This is particularly true in patients with previously untreated minimally classic and occult lesions in whom VA improvement and lesion size stabilization has been recorded. These results are very important and contrast with the relatively moderate treatment effects seen in minimally classic and occult lesion after PDT.

In our patients, a frequent discrepancy was also observed between anatomic factors, examined with fluorescein angiography (FAG) and indocyanine green angiography (ICGA), and functional factors: VA improvement is often not correlated 
with lesion size changes in FAG+ICGA. Our observations have also led us to conclude that pegaptanib sodium has a slower mode of action compared with that of unselective VEGF inhibitors. Clinical experience would suggest that, because pegaptanib sodium's mode of action is slower, at least 3 ITV injections are required to produce good efficacy.

In a recent study, Quiran et al evaluated the efficacy of pegaptanib sodium as primary therapy in 90 patients with naive lesions ( $80 \%$ occult, $13 \%$ minimally classic, and $7 \%$ predominantly classic) (Quiran et al 2007). All patients were injected with $0.3 \mathrm{mg}$ of ITV pegaptanib sodium at 6-week intervals. FAG and OCT were carried out at baseline and were repeated after 3 treatments, whereafter an additional ITV injection of pegaptanib sodium was administered if the CNV was observed to be active. A response rate of $90 \%$ was recorded with: $20 \%$ of patients gaining $\geq 3$ lines of vision; vision stabilization being achieved in $70 \%$ of patients, and a loss of $\geq 3$ lines being recorded in $10 \%$ of patients. An average of 3.5 injections were administered in those patients who experienced a gain in vision of $\geq 3$ lines.

Our experience echoes the findings of Quiran et al in that we also found 3-4 injections to be enough to stabilize the lesion size and to either stabilize or improve VA in the majority of patients with early lesions. One occult lesion treated with pegaptanib sodium is described in the following case study.

An 82-year-old woman was referred to our department after a loss of VA in her right eye (RE). Best corrected VA
(BCVA) was 10/20 in her RE and 18/20 in her left eye. Biomiscropy of the anterior segment was unremarkable in both eyes. FAG+ICGA and OCT revealed an occult CNV membrane associated with neovascular AMD in the RE (see Figure 1). The patient was treated with 5 ITV injections of of pegaptanib sodium given 6-weekly.

FAG+ICGA and OCT were performed before every injection. The patient's RE VA stabilized after 2 injections and FA+ICGA showed an increase in lesion leakage. At follow-up after the third injection, VA in the RE had improved (16/20) and the lesion size was stable (as assessed with either with FAG+ICGA and OCT). One month after the fifth ITV injection, both VA and lesion size were stable and treatment was stopped. At follow-up 9 months after last injection, BCVA was 16/20 and FAG+ICGA and OCT showed an absence of lesion activity (see Figure 1).

Stable VA is commonly seen after initial injections with selective anti-VEGF treatment, but lesion activity often persists on angiography. As the above clinical case exemplifies, however, VA and the angiographic pattern in the discussed patient were stable after only 2 injections of pegaptanib sodium and the beneficial effects of treatment were evident after only 3 treatments.

From our clinical experience, we conclude that at this time pegaptanib sodium has good efficacy and safety profiles and represents a good treatment option for patients with early CNV membranes associated with neovascular AMD.

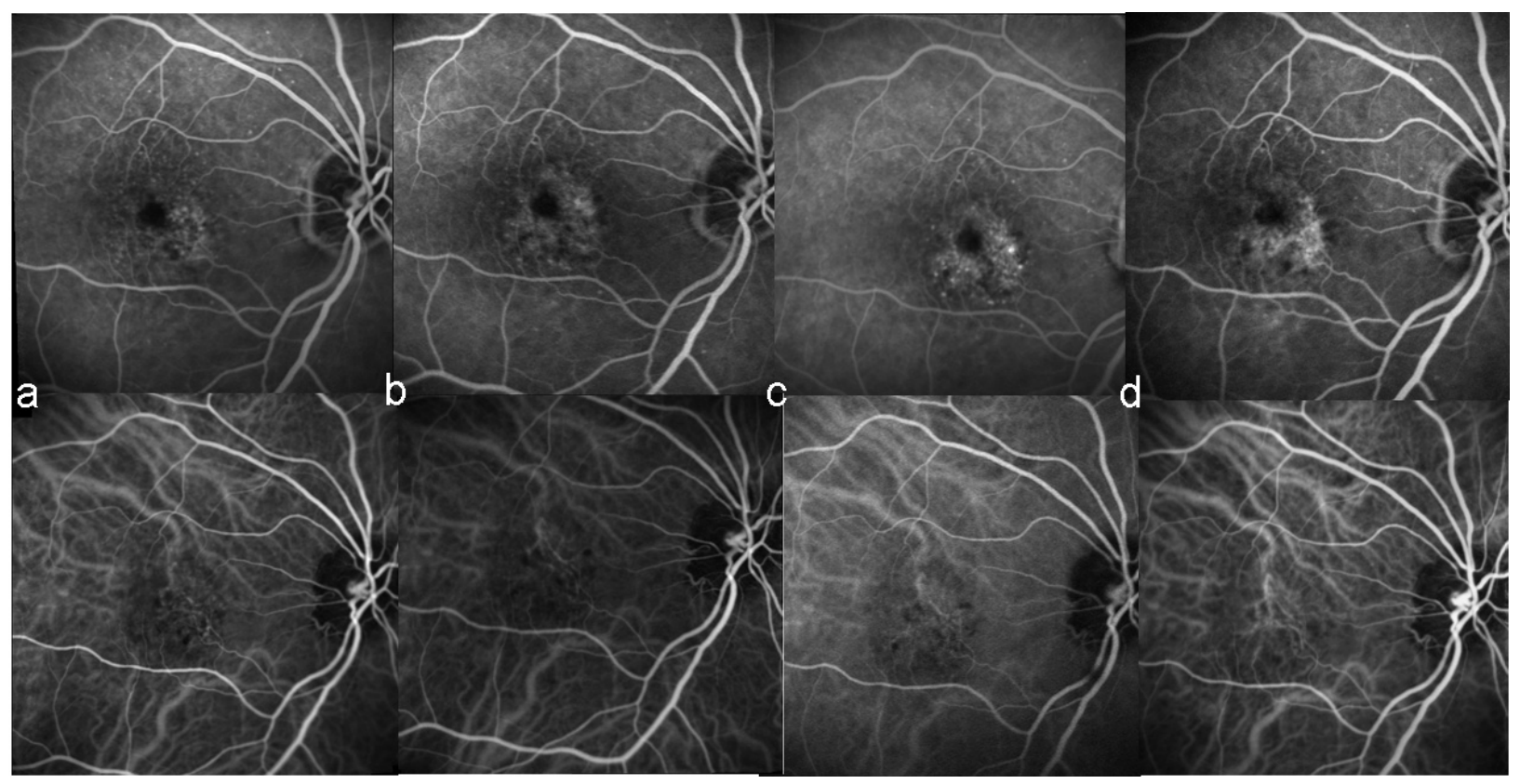

Figure I Fluorescein angiography (FAG) and indocyanine green angiography (ICGA) from an 82-year-old female patient after loss of visual acuity (VA) in her right eye (RE) and who received 5 injections of intravitreal (ITV) pegaptanib sodium.

a. pre-injection; b. post second injection; c. post third injection; d. 9 months post fifth injection. 


\section{Disclosures}

Prof Staurenghi has received research grant support from Novartis, Pfizer, and Allergan. Drs Rosina and Bottoni have no conflicts of interest to disclose.

\section{References}

Brown DM, Kaiser PK, Michels M, et al; ANCHOR Study Group. 2006. Ranibizumab versus verteporfin for neovascular age-related macular degeneration. $N$ Engl J Med, 355:1432-44.

Chakravarthy U, Adamis AP, Cunningham ET Jr, et al; VEGF Inhibition Study in Ocular Neovascularization (V.I.S.I.O.N.) Clinical Trial Group. 2006. Year 2 efficacy results of 2 randomized controlled clinical trials of pegaptanib for neovascular age-related macular degeneration. Ophthalmology, 113:1508 e1-25.

D'Amico DJ, Masonson HN, Patel M, et al; VEGF Inhibition Study in Ocular Neovascularization (VISION.) Clinical Trial Group. 2006. Pegaptanib sodium for neovascular age-related macular degeneration: two-year safety results of the two prospective, multicenter, controlled clinical trials. Ophthalmology, 113:992-1001.

Gonzales CR; VEGF Inhibition Study in Ocular Neovascularization (V.I.S.I.O.N.) Clinical Trial Group. 2005. Enhanced efficacy associated with early treatment of neovascular age-related macular degeneration with pegaptanib sodium: an exploratory analysis. Retina, 25:815-27.
Gragoudas ES, Adamis AP, Cunningham ET Jr, et al; VEGF Inhibition Study in Ocular Neovascularization (V.I.S.I.O.N.) Clinical Trial Group. 2004. Pegaptanib for neovascular age-related macular degeneration. N Engl J Med, 351:2805-16.

McDermott MP, Hall WJ, Oakes D, et al. 2002. Design and analysis of two- period studies of potentially disease-modifying treatments. Control Clin Trials, 23:635-49.

Mills E, Hells-Ansdell D, Kelly S, et al. 2007. A randomized trial of pegaptanib sodium for age-related macular degeneration used an innovative design to explore disease-modifying effects. J Clin Epidemiol, 60:456-60.

Quiram P, Hassan TS, Williams GA. 2007. Treatment of naive lesions in neovascular age-related macular degeneration with pegaptanib. Retina, 27:851-6.

Rosenfeld PJ, Brown DM, Heier JS, et al; MARINA Study Group. 2006a. Ranibizumab for neovascular age-related macular degeneration. $N E n g l$ $J$ Med, 355:1419-31.

Rosenfeld PJ, Rich RM, Lalwani GA. 2006b. Ranibizumab: Phase III clinical trial results. Ophthalmol Clin North Am, 19:361-72.

Whitehouse PJ, Kittner B, Roessner M, et al. 1998. Clinical trial designs for demostrating disease-course-altering effects in dementia. Alzheimer Dis Assoc Disord, 12:281-4. 